\title{
El presupuesto participativo como fortalecimiento de la democracia.
}

\author{
Luis Miguel Villareal Aguilar ${ }^{a} \&$ Jaime Bermejo Galán ${ }^{\mathrm{b}}$ \\ ${ }^{a}$ Facultad de Ciencias Exactas, Universidad de Cartagena, Cartagena, Colombia. \\ lvillareala@unicartagena.edu.co. \\ ${ }^{b}$ Facultad de Ciencias Jurídicas y Sociales, Universidad Simón Bolívar, Barranquilla, Colombia. \\ jbermejo@unisimonbolivar.edu.co
}

Recibido: Noviembre 26, 2020.

Recibido en su versión corregida: Enero 22, 2021.

https://doi.org/10.54606/Sextante2021.v24.06

Aceptación: Mayo 12, 2021.

Cómo citar: Villareal Aguilar, L.M. \& Bermejo Galán, J. (2021) El presupuesto participativo como fortalecimiento de la democracia. Revista Sextante, 24, pp. 44 - 55, 2021.

\section{Resumen}

El Presupuesto Participativo es una herramienta complementaria de los procesos de planificación participativa desarrollados en nuestro país. En este sentido se comprende como una estrategia de organización y movilización social y un proceso pedagógico que apunta al fortalecimiento de las organizaciones sociales respecto a su ejercicio político, en donde la participación con decisión debe reconocerse como un derecho fundamental de la ciudadanía. No obstante, el Presupuesto Participativo contemplado en los planes de desarrollo distrital, y la buena ejecución de este, no se da, puesto que se gestionan de forma improcedente los procesos participativos, omitiendo así la convocatoria que se le debe hacer a la ciudadanía para que ejerza su derecho de participación, a pesar de lo que se determina en la Ley 1551 de 2012.

Palabras clave: Democracia participativa; Juntas de acción comunal; Participación ciudadana; Presupuesto participativo.

\begin{abstract}
The Participatory Budget is a complementary tool to the participatory planning processes developed in our country. In this sense, it is understood as a strategy of the organization and social mobilization and a pedagogical process that aims to strengthen social organizations concerning their political exercise. Participation in the decision must be recognized as a fundamental right of citizenship. However, the Participatory budget contemplated in the district development plans, and its proper execution, does not occur, since the participatory processes are improperly managed, thus omitting the call that must be made to the citizens so that exercise your right of participation, despite what is determined in Law 1551 of 2012.
\end{abstract}

The participatory budget as a strengthening of democracy.

Keywords: Citizen Participation; Community action coards; Participatory budget; Participatory democracy. 


\section{Introducción}

El presente documento busca analizar el proceso de implementación del presupuesto participativo en la ciudad de Cartagena, en el periodo comprendido entre 2016-2019, entendiendo que este es una herramienta innovadora en comparación al enfoque tradicional de la planificación distrital. Para dicho proceso se tienen en cuenta dos actores principales, el Gobierno Distrital y los ciudadanos, quienes se encargan de tener un diálogo deliberativo y participativo, donde al final prima la toma de decisiones y la cogestión de una parte del presupuesto de la ciudad. Los habitantes del territorio son los protagonistas de este proceso, puesto que serán los que determinen las prioridades de obra e inversión pública para sus entornos territoriales (Localidades).

En esa medida, se han planteado cuatro capítulos, a través de los cuales se realizará el análisis propuesto, para empezar se realizará un recorrido por la normativa que cobija a la herramienta del presupuesto participativo en Colombia, posterior se examinará la experiencia de este proceso en Bogotá y se realizará una comparación con la de Cartagena, luego seguirá la identificación de los obstáculos que posee la implementación del presupuesto participativo en la ciudad de la región Caribe, y finalmente, se propondrá una estrategia que propenda por resolver parte de las problemáticas encontradas; y al mismo tiempo genere verdaderos espacios de encuentro y participación entre la administración distrital y la ciudadanía.

\section{Planteamiento del problema}

Hablar de Presupuesto Participativo (PP) implica examinar la literatura de distintos autores; entre estos se encuentran Pagliai y Montecinos (2006), quienes afirman que éste es una herramienta de planificación local que permite a los territorios involucrar a sus habitantes a través de un proceso de información, deliberación, decisión y control sobre la inversión de los recursos, teniendo en cuenta que esto posibilita la priorización de las demandas ciudadanas, facultando a la población en la toma de las decisiones públicas. Algo semejante explica Urán (2007), quien concibe el Presupuesto Participativo como un espacio de cogestión donde los territorios, en conjunto con sus ciudadanos, deciden la distribución de una parte de las inversiones. Finalmente está Betancourt (2005), quien aduce que el PP tiene como propósito optimizar la redistribución de la riqueza al posibilitar la participación democrática de los habitantes en las decisiones que tienen que ver con el presupuesto público.

En tal sentido, a partir de las definiciones anteriores se puede deducir que el presupuesto participativo es una estrategia que envuelve dos términos, la gobernabilidad y la democracia. En primer lugar, porque es una metodología que vincula a actores diferentes a las autoridades gubernamentales en las decisiones, en este caso la comunidad; $y$, en segundo lugar, porque todo este proceso que se enmarca en la democracia se encamina hacia una cultura participativa y a una gestión transparente y eficiente.

Desde esta perspectiva el Estado está compelido a construir y ejecutar los presupuestos de manera que se genere una mayor cohesión política de los ciudadanos con sus autoridades locales, y permita que la ciudadanía, en pleno reconocimiento de sus derechos, ejerza la participación en las decisiones sobre el destino de los recursos en el territorio.

No obstante, la realidad dista de la teoría. Si bien, el Presupuesto Participativo se contempla en los planes de desarrollo distrital, la buena ejecución del mismo, no se da, puesto que se gestionan y emprenden de forma improcedente los procesos participativos, omitiendo así la convocatoria que se le debe hacer a la ciudadanía para que ejerza su derecho de participación, opine y discuta sobre: 1) la programación de proyectos; 2) el orden de priorización en el cual se deberían ejecutar; y 3 ) la veeduría de su cumplimiento, a pesar de lo que se determina en la Ley 1551 de 2012, la cual afirma que "Los municipios gozan de autonomía para la gestión de sus intereses, dentro de los límites de la Constitución y la ley" (Congreso de la República de Colombia, 2012, p.1).

Cabe resaltar que estas prácticas participativas abarcan un amplio espectro a nivel territorial, sectorial y poblacional. Han sido implantadas, simplemente, para dar soporte a lo estipulado en la Constitución Política y sus respectivas reglamentaciones, ignorando el aspecto ético y conceptual; realizando hincapié en su acción instrumental (Contreras 2007). Ahora bien, se deja 
de manera clara que dichas herramientas de participación, como lo es el Presupuesto Participativo, causan controversia debido a la efectividad de estas en cuanto a resultados. Tal cual lo afirma Subirats (2005) cuando dice que este tipo de metodologías son más propensas a que tengan una escasa efectividad para acercar las preferencias de los ciudadanos a las soluciones públicas, porque las dinámicas que implican son diversas y dependen del escenario en el cual se implementen.

En esa medida, hablar de planeación se vuelve esencial, ésta es de los activos más importantes al momento de discutir la destinación de los recursos, en aras de logar los objetivos que tienen los programas de los gobiernos locales. Su papel es el de obtener un equilibrio dinámico y enriquecedor entre la visión de conjunto, es decir, entre lo que aporta la ciudadanía y lo que aportan las instituciones gubernamentales (Jiménez et al, 2013).

Esta actividad de síntesis es vital para organizar y ejecutar los distintos programas de gobierno desde una visión estratégica, puesto que es el resultado de la gestión local donde se trabaja en equipo para sacar las iniciativas resultantes de una planeación conjunta, teniendo en cuenta las necesidades de los habitantes y sus localidades.

Hay que tener en cuenta que el Presupuesto Participativo es una herramienta relativamente nueva en Colombia y Latinoamérica. Uno de los pioneros en el tema fue Porto Alegre (Brasil). La experiencia de este país y su éxito se basa en dos grandes aspectos: en primer lugar, uno de sus propósitos principales era privilegiar la participación ciudadana; y en segundo lugar, su fin era, también, precisar un modelo de gestión político administrativa en donde el énfasis estuviera en la gestión de los recursos públicos y las demandas atendidas de los ciudadanos (Becerra, 2017). Si bien, el caso de Brasil dio buenos resultados y fue tomado como ejemplo para replicar su metodología, también hizo explícitas las dificultades que este proceso presentó. Recordemos que fue desde 1989 que comenzó el experimento, y sólo hasta el año 2002 , se comenzaron a visibilizar los resultados y la superación de las dificultades.

Sánchez (2004) señala que, en las experiencias a lo largo de toda Latinoamérica, regularmente se encuentran tres dificultades "comunes" en función de los actores que están involucrados en el proceso del presupuesto participativo.

En primer lugar, hace referencia a los actores políticos. Esta metodología requiere compartir el poder de decisión, es decir, los gobiernos deben ceder parte del poder a la ciudadanía, sin que esto signifique liberarse totalmente de la responsabilidad que como ente gubernamental le compete. En esta medida se requiere de una gran voluntad política para llevar a cabo el proceso.

En segundo lugar, existe el aspecto técnico, hablando en términos de planificación técnica, realizar un PP requiere un ajuste a los tiempos y ritmos de la participación ciudadana, pues son estos quienes establecen prioridades, de esa manera el proceso puede ser dilatado a causa de la baja participación que se presenta en los territorios.

Finalmente, se encuentra la tercera dificulta, que hace alusión a las organizaciones sociales, pero más que todo a la representatividad de los ciudadanos. Distribuir la representatividad puede ser algo complejo y como ya se mencionó anteriormente, esta metodología está basada en la toma de decisiones dadas en el proceso de discusión colectivo, en ese orden de ideas, se hace "necesaria la participación e implicación de las organizaciones, colectivos sociales y habitantes, como canalizadores de la participación ciudadana y conocedores de la realidad social de los territorios o las temáticas que se abordan”. (Sánchez, 2004, p. 335)

Lo anterior, sumado a la desmotivación de la población para participar en las asambleas de las comunidades; la insuficiencia de conciencia política de la necesidad de participación y del ejercicio de ciudadanía; la difusión limitada y desinformación en la comunidad; y la falta de recursos económicos para cubrir todos los proyectos y obras priorizadas, son los obstáculos que muestran una realidad que puede ser utilizada como espejo al momento de reparar el proceso de implementación del Presupuesto Participativo en Cartagena de Indias, esto entre otros factores que se han hecho evidentes a lo largo de los años.

En ese orden de ideas, la presente investigación examina toda la problemática y los aspectos que se han presentado en los últimos 4 años para la implementación del Presupuesto Participativo en Cartagena. 


\section{Marco jurídico del Presupuesto Participativo}

En aras de realizar la descripción del marco legal, que aborda la implementación de la herramienta de los presupuestos participativos en Cartagena, primeramente, se debe hacer la salvedad de que no existe una ley específica y detallada que regule el tema del PP en Colombia. A pesar de esto, este proceso se acobija bajo distintas normativas que tienen que ver con la planeación y régimen presupuestal.

En primer lugar, se encuentra la Ley 1551 de 2012, que estipula reglas para modernizar la normativa asociada con el régimen municipal, dentro de la autonomía que reconoce a los municipios la Constitución y la ley, como instrumento de gestión para cumplir sus competencias y funciones. De igual manera, en su artículo 32, ésta determina la opción de que los municipios puedan crear ejercicios de presupuestación participativa (Congreso de la República de Colombia, 2012).

Posterior a esto, se expide la Ley Estatutaria 1757 de 2015, ella tiene como objetivo "promover, proteger y garantizar modalidades del derecho a participar en la vida política, administrativa, económica, social y cultural, y así mismo a controlar el poder político" (Congreso de la República de Colombia, 2015, p.1). Esta última norma es la primera que hace alusión de manera directa al tema del Presupuesto Participativo,

Dicha norma expresa en su artículo 100 lo siguiente: Los gobiernos de los entes territoriales previstos en la Constitución y la ley podrán realizar ejercicios de presupuesto participativo, en los que se defina de manera participativa la orientación de un porcentaje de los ingresos municipales que las autoridades correspondientes definirán autónomamente, en consonancia con los objetivos y metas del Plan de Desarrollo. (Congreso de la República de Colombia, 2015, p.29)

Dentro de este tema del PP se discute sobre dos lógicas: 1) Lógica de justicia política: en la cual todas las personas tienen derecho a participar en decisiones que las afectan de manera directa o indirecta; y 2) Lógica de eficacia: es decir, que, si la ciudadanía puede participar en la toma de decisiones sobre las problemáticas que aquejan su realidad, las soluciones que se generen serán más relevantes y apropiadas. Significaría una disminución en el despilfarro de los recursos y se les daría un mejor uso. Para tener un poco más de claridad sobre el marco legal del PP en la Tabla 1 se encuentran las normas relacionadas con la planeación y el presupuesto participativo.

Ahora bien, para el caso específico de Cartagena, la norma que lo regula y lo pone en el mapa político de la ciudad es el Acuerdo Distrital 003 del 20 de marzo de 2009, por el cual se institucionaliza el proceso de presupuesto participativo, como sistema de fortalecimiento y desarrollo del sistema distrital de planeación y se dictan otras disposiciones. Lo estipulado en este acuerdo alude a lo imperante de la participación ciudadana en el proceso de construcción del presupuesto participativo, y que es de vital importancia que se realice una inscripción de los distintos delegados de la ciudad, tanto de habitantes de Cartagena en general, como de organizaciones sociales, comunitarias y solidarias.

De esta manera se impone en la norma que: "los habitantes del Distrito de Cartagena de Indias intervienen en el proceso de Presupuesto Participativo a través de la elaboración de propuestas para el presupuesto de la localidad en donde viven y para el presupuesto general, respecto a aquellas políticas sectoriales que involucran al conjunto de la Ciudad" (Concejo Distrital Cartagena de Indias, 2009). Reafirmando una vez más el papel fundamental que cumplen los ciudadanos en la metodología e implementación de esta herramienta.

Los mecanismos de participación ciudadana y comunitaria se han promulgado de manera diversa, ya sea mediante leyes o acuerdos, como se ha dicho anteriormente, pero su historia data de lo reglamentado en los derechos fundamentales de la Constitución Política de Colombia de 1991. A partir de estos se hace posible entonces, que los ciudadanos del territorio accedan a la información sobre los planes y programas que ejecuta el gobierno local, así como también los faculta en la toma de decisiones alrededor de proyectos que propendan por el desarrollo del municipio, y de igual forma realizar veeduría a la administración municipal.

Después de este breve recorrido, en donde se realizó la descripción del marco jurídico que susten- 
Villareal Aguilar \& Bermejo Galán. (2021). Revista Sextante, 24, pp. 44 - 55, 2021.

Tabla 1. Normas relacionadas con la planeación y el presupuesto participativo.

Normas Relacionadas con la Planeación y el Presupuesto Participativo

\begin{tabular}{lll}
\hline \multicolumn{1}{c}{ Tema } & \multicolumn{1}{c}{ Norma } & \multicolumn{1}{c}{ Texto } \\
\hline Acuerdos & Ley 1757 de 2015, & El proceso del presupuesto participativo es un mecanismo de \\
participativos & Art. $90(100)$ & $\begin{array}{l}\text { asignación equitativa, racional, eficiente, eficaz y transparente } \\
\text { de los recursos públicos, que fortalece las relaciones Estado - } \\
\text { Sociedad Civil. Para ello, los gobiernos regionales y locales } \\
\text { promueven el desarrollo de mecanismos y estrategias de } \\
\text { participación en la programación de sus presupuestos, así como } \\
\text { en la vigilancia y fiscalización de la gestión de los recursos } \\
\text { públicos. }\end{array}$ \\
& & $\begin{array}{l}\text { Para efectos de cumplimiento de la presente ley, las Asambleas } \\
\text { Ley 1757 de 2015, Concejos Municipales y Distritales, y las Juntas }\end{array}$ \\
& Parágrafo Art. 93 & Administradoras Locales, deberán incorporar los acuerdos \\
& participativos previos a la discusión de los presupuestos \\
& plurianuales.
\end{tabular}

\begin{tabular}{ll}
\hline Presupuesto & C.P. Art. 345, 346, 347, \\
& 348, \\
& $349,350,351,352,353$.
\end{tabular}

Requisitos para el Ley 358 de $1997 \quad$ Establece los indicadores y requisitos para que el municipio, endeudamiento distrito o departamento pueda solicitar endeudamiento.

Ley de ajuste fiscal Ley 617 de $2000 \quad$ Se conoce como de responsabilidad fiscal, donde se definen límites al gasto de funcionamiento de acuerdo con los ingresos propios del municipio, distrito o departamento.

Sistema general de Ley 715 de 2001

Participaciones
Allí se establecen las transferencias de la nación hacia los departamentos, municipios y distritos, según las competencias de cada ente territorial en la presentación y financiación de los servicios y derechos básicos de educación, salud, alimentación escolar y saneamiento básico.
Planeación del C.P. Art. 339 desarrollo
"Las autoridades territoriales elaborarán y adoptarán, de manera concertada entre ellas y el Gobierno Nacional, planes de desarrollo, con el objeto de asegurar el uso eficiente de sus recursos y el desempeño adecuado de las funciones que le hayan sido asignadas por la Constitución y la Ley.

Ley 152 de 1994

o Ley 388 de 1997

(de orden territorial)
La Ley 152 considera la participación ciudadana como central, particularmente en la instancia de los Consejos Territoriales de Planeación, que son un ámbito clave en el proceso de discusión del Plan de Desarrollo. Este espacio está conformado por personas designadas por el Gobernador o el alcalde, de las ternas que presenten las correspondientes autoridades y organizaciones de acuerdo con la composición que definan las Asambleas o Consejos. En los Consejos Territoriales deberán estar representantes de los sectores económicos, sociales, ecológicos, educativos, culturales y comunitarios. (Artículo 340 de la Constitución) es decir, que los Consejos deben contener la representación de los diversos sectores de la sociedad civil. 
-ta el PP, se puede deducir que hay una disposición legal explícita, que abarca la implementación del Presupuesto Participativo en la ciudad de Cartagena, con unas normas direccionadas a aumentar la participación y transparencia de la gestión pública; y la asignación y control de los recursos.

\section{Capítulo 1: Examinar la experiencia de la} construcción del Presupuesto Participativo en la ciudad de Bogotá en comparación a la experiencia en la ciudad de Cartagena de Indias.

Colombia es un país administrativamente descentralizado, y si bien cada departamento y municipio son, en cierta medida, autónomos en cuanto a su progreso y desarrollo, es importante ahondar en la forma sobre cómo está llevando a cabo el proceso del presupuesto participativo la capital del país, las estrategias y bajo qué modelo se está implementando dicha herramienta. Bogotá comenzó a ejecutar esta metodología participativa en el periodo de 1995-1998, cuando la administración local convocó de manera directa a las organizaciones y comunidades a presentar sus proyectos con un comité técnico local para la planeación, pero no fue hasta el periodo de 19982001 que se realizaron los encuentros ciudadanos para acordar el contenido del plan de desarrollo (Becerra, 2017).

El proceso fue avanzando y se adelantaron distintas experiencias, por ejemplo: en el periodo de 2001 al 2004 se constituyeron los Consejos de Planeación Local; en 2011 se visibilizó un poco más la materialización de esto con la formulación de del decreto 503 del mismo año, a través del cual se adoptó la política pública de participación para el Distrito Capital. En el periodo de 2012-2106 se tuvo una experiencia más completa, con un desarrollo propiamente participativo.

Cuando se crea el plan de desarrollo 'Bogotá Humana 2012-2016', la participación ciudadana entra a jugar un papel fundamental en la toma de decisiones de la gestión pública, puesto que en su artículo 36 se disponía que uno de los proyectos con mayor priorización de este programa sería el de Planeación y presupuesto participativo, para la superación de la segregación y discriminación social, económica, espacial y cultural (Alcaldía Mayor de Bogotá, 2012). El accionar de esto fue bastante rápido, la administración delimitó la ciudad en 188 territorios para su implementación, y de estos se seleccionaron 8 unidades básicas de participación para realizar una prueba piloto de esta herramienta.

El proceso se dividió en 5 fases: 1) La definición de insumos y presupuesto; 2) La convocatoria, sensibilización y formación; 3) El proceso deliberativo y de priorización; 4) Los procesos decisorios e inclusión de iniciativas; y 5) La evaluación. La metodología fue construida por la administración del momento, así como también fueron los encargados de establecer los montos de inversión. En el 2012 se pudieron materializar los resultados de este trabajo, el cual desembocó en la priorización de las iniciativas depuradas en los cabildos de gobernanza del agua, juventud y malla vial. (González, 2012)

Según Tabares (2014), de las anteriores experiencias se puede evidenciar que en cada una de las unidades que hicieron parte del piloto, se llevaron a cabo las 5 etapas en su totalidad. Es decir, cada una de las unidades pasó por un proceso donde se expusieron las problemáticas que los afectaban, luego por otro en donde se deliberaron las mismas en aras de validarlas, y por último, un acto de toma de decisión en cuanto a la priorización de dichas problemáticas, para así asignar los recursos correspondientes y solventarlas. De igual forma, se denotó un proceso de participación ciudadana apegado a la norma, como lo fue el caso del estatuto orgánico y del plan de desarrollo Bogotá Humana. Finalmente, se materializó el poder de decisión de la comunidad al momento en que estos tuvieron la facultad de decidir sobre cómo invertir recursos en sus localidades según sus necesidades, teniendo estos un peso importante en el presupuesto de cada una de ellas.

En ese sentido, es claro que en Bogotá la metodología del Presupuesto Participativo va adelantada, pues a través de los años se han venido desarrollando diversas experiencias que apuntan al tema. Ese no es el caso de la ciudad de Cartagena. El primer intento de desarrollar este tipo de procesos en La Heroica fue en el periodo 2016-2019 con el plan de desarrollo distrital 'Primero la gente', en donde se estipuló el programa Presupuesto Participativo, el cual buscaba generar los espacios de participación ciudadana para la toma de decisiones que posibilitaran priorizar las acciones del gobierno local, teniendo en cuenta las necesidades identificadas por la misma comunidad, 
y así ejecutar el proceso del PP en concordancia a lo determinado en el acuerdo 003 de 2009 y su decreto reglamentario 0761 de 2009. (Alcaldía de Cartagena, 2016)

Tal proceso tuvo sus obstáculos pues, a pesar de estar constituido en el Plan de Desarrollo, las acciones llevadas a cabo no se realizaron. En una revisión documental realizada al plan de acción de la secretaria del interior en el 2015 y 2016, se encontró que no se ejecutó ninguna asamblea popular cuando se tenían planificadas 30 asambleas populares, una por cada Unidad Comunera de Gobierno del distrito, en aras de deliberar las problemáticas, establecer la priorización de estas y la inscripción de proyectos al banco de proyectos, a partir de lo concluido en tales asambleas. De esa manera se visibiliza que el caso Cartagena en contraposición de la experiencia de Bogotá no fue del todo exitoso (Secretaria del Interior Distrital, 2016).

\section{Capítulo 2: Identificar la problemática relacionada con la implementación de instrumentos relacionados a la Gestión Participativa en la Ciudad de Cartagena de Indias.}

El tema de la gestión participativa en Colombia tuvo su auge en año 1991 con la nueva Constitución Política, esto se dio de manera natural, a causa del clima político que se vivía en el país para esa época, y de esa forma se instauró la participación como un derecho fundamental. Así que, en aras de dar validez a su nuevo propósito: 'la construcción de un nuevo país', se decide involucrar a los distintos actores que se vieron envueltos en la lucha por este proceso, como lo eran los jóvenes universitarios, intelectuales, miembros de las O.N.G., y gente que para en ese entonces era proveniente de varios proyectos de la izquierda radical (Borda, 1998).

Posterior a esto fue reglamentada la democracia directa y participativa con la Ley 134 de 1994, que exponía "los mecanismos de participación del pueblo regulan la iniciativa popular legislativa y normativa; el referendo; la consulta Popular, del orden nacional, departamental, distrital, municipal y

\footnotetext{
${ }^{1}$ Dada la dificultad de contar con estudios empíricos para diferentes municipios nos vamos a referir fundamentalmente a la ciudad de Medellín: ver: Fernández A. Rubén et al "Visión
}

local; la revocatoria del mandato; el plebiscito y el cabildo abierto" (Congreso de la República de Colombia, 1994, p.1). Se denota entonces que este fue el comienzo de la participación al crear los distintos mecanismos que permitirían a la ciudadanía tomar parte en las decisiones del gobierno.

La misma también estipula la creación de dos esferas de acción ciudadana; la propiamente política y la de gestión institucional. La primera hace alusión a las maneras de incidir en el poder institucional mediante las diversas figuras de democracia directa, es decir, los mecanismos de participación anteriormente mencionados. Esta intención de incidir en el poder institucional también se formuló a través de una combinación entre democracia directa y descentralizada, direccionada hacia la vida regional y local a través de mecanismos como la revocatoria del mandato de gobernadores y alcaldes, la vigilancia y el control de las instancias públicas y la elaboración de planes de desarrollo (García Villegas, 2003).

Por otro lado, está el aspecto de la gestión pública, la cual hace referencia a iniciativas amplias y complicadas, que tocan los ámbitos desde la participación en las juntas directivas de las empresas prestadoras de servicios públicos y sociales, hasta la conformación de las Juntas Administradoras Locales (JAL), señalando una diversidad de espacios y actividades creadas, de carácter distinto y asociado con la implementación de políticas sectoriales estipuladas para hacia los jóvenes, mujeres, grupos étnicos subordinados, trabajadores y campesinos. Se vuelve necesario entonces decir que la mayoría de las veces, los gobiernos toman acción y brindan espacios de participación a la ciudadanía, más por cumplir lo que dice la ley que por realizar un verdadero ejercicio de aproximación a la organización social ${ }^{1}$.

Teniendo en mente el objetivo principal de la ejecución de los presupuestos participativos: la potenciación de una democracia participativa, permitiendo a la ciudadanía el proceso de toma de decisiones (Mairal, 1998). Se decidió buscar la forma de generar la participación de forma directa

Panorámica de la Participación Ciudadana en la Ciudad de Medellín”. Corporación Región. 2000 (s.e.) Mimeo. P4 
de los habitantes del territorio en la construcción del presupuesto municipal, haciendo énfasis en la priorización de proyectos y programas que tuvieran afinidad con las problemáticas que manifestaban los ciudadanos. Es aquí cuando se empezaron a denotar los obstáculos que acarrea la implementación de instrumentos asociados a la gestión participativa, como es el caso del presente documento, el PP.

\section{Artículo 4. - Asambleas populares y comunitarias.}

El proceso de presupuesto participativo para las Unidades Comuneras de Gobierno se implementa a través de Asambleas Populares Comunitarias, en las que los habitantes de cada una de ellas discuten, elaboran propuestas y definen prioridades para su presupuesto. Así mismo realizan el seguimiento y control del presupuesto en ejecución, todo sobre la base de la información que trimestralmente les remite la Secretaria de Planeación del distrito de Cartagena de Indias (Concejo Distrital de Cartagena de Indias, 2009).

Habiendo claridad de la metodología para llevar a cabo el presupuesto participativo en la ciudad, se procedió a explorar el plan de desarrollo 2020-2023 'Salvemos Juntos a Cartagena', que se encuentra dado como una estrategia de participación y descentralización. Esta plantea el fortalecimiento de la participación ciudadana y comunitaria como principio constitucional que reconozca a los ciudadanos como partes activas en la toma de decisiones de carácter institucional, social y comunitario que puedan afectarlos (Alcaldía de Cartagena, 2020). En esa medida, al interior de la línea, está contemplado un programa denominado Presupuesto Participativo, que propone la priorización de la inversión por parte las comunidades, ajustada a los procedimientos normativos para tal fin.

En la Tabla 2 se presentan los logros que traza el plan de desarrollo distrital respecto este tema.

Se puede observar, en primer lugar, que la última priorización fue realizada en 2009 y la meta para este periodo es realizar una priorización de proyectos del PP. En este sentido no dista de la meta propuesta para el periodo 2016-2019, puesto que es la misma. No obstante, la diferencia se encuentra en la ejecución de los proyectos priorizados. Mientras que para el 2016-2019 la meta era ejecutar la totalidad de los nuevos proyectos priorizados, la meta para el periodo actual es ejecutar 30 proyectos priorizados.

En un principio, para la época del plan de desarrollo 'Primero la gente' los impedimentos para llevar a cabo el presupuesto participativo radicaban en la falta de voluntad política para realizar las asambleas que determinarían el destino de los recursos, pues como se mencionó, en el plan de acción de la secretaria del interior del 2015 se había definido la realización de 30 asambleas populares con un presupuesto fijo de $\$ 613.854 .808$, el cual abarcaba a toda la ciudad, no obstante, no se ejecutó ninguna asamblea, y para el año 2016, sucedió algo similar, aun cuando se tenía un presupuesto estimado de \$ 800.000.000 (Secretaria del Interior Distrital, 2016).

Ahora el problema radica en la falta de presupuesto. Al hacer la revisión del plan de acción 2020 y 2021 de la Secretaria del Interior Distrital, se observa que no hay ninguna actividad estipulada para el proyecto de Presupuesto Participativo, entre las razones se encuentra que para el 2020 no se le asignaron recursos en el plan plurianual de inversiones y que para el 2021 en el Acuerdo No. 044 de fecha 18/12/2020 y decreto de liquidación de presupuesto $\mathrm{N}^{\circ} 1637$ de 29/12/2020, no se le asignaron recursos al programa de presupuesto participativo para la vigencia actual (Secretaria del Interior Distrital, 2020-2021).

De tal manera, se concluye que los impedimentos para la implementación de la gestión participativa, de manera específica, en cuanto a la herramienta de presupuesto participativo, se basa en dos aspectos.

El primero es la falta de voluntad política por parte de los mandatarios de tuno, al no querer cumplir con lo establecido en sus planes de gobierno, y más importante, con lo estipulado en la ley; esto sumado a la carencia de procesos planificados, procesos de capacitación permanente; veracidad y confiabilidad en las convocatorias; suministro de información permanente, actualizada, y veraz. El segundo, y considerado como factor actual, es la no determinación de recursos públicos, para la financiación, y llevar a cabo la logística que una metodología participativa como esta requiere. 
Tabla 2. Programa: Presupuesto Participativo - Plan de Desarrollo Distrital 'Salvemos Juntos a Cartagena' 2020-2023.

\begin{tabular}{|c|c|c|c|}
\hline Indicador de producto & Linea base & Meta 2020-2023 & Responsables \\
\hline $\begin{array}{l}\text { Número de priorizaciones de } \\
\text { proyectos de presupuesto } \\
\text { realizadas. }\end{array}$ & $\begin{array}{l}\text { Última priorización } \\
\text { realizada en } 2009 . \\
\text { Fuente: Secretaría del } \\
\text { interior y convivencia } \\
\text { ciudadana. }\end{array}$ & $\begin{array}{l}\text { Realizar una priorización de proyectos } \\
\text { de presupuesto participativo en cada } \\
\text { una de las UCG urbanas y rurales en el } \\
\text { Distrito de Cartagena. }\end{array}$ & $\begin{array}{l}\text { Secretaría del Interior y } \\
\text { Convivencia } \\
\text { Ciudadana. }\end{array}$ \\
\hline $\begin{array}{l}\text { Números de } \\
\text { presupuectos por } \\
\text { ejecutados. }\end{array}$ & ND & $\begin{array}{l}\text { Ejecutar } 30 \text { proyectos priorizados por } \\
\text { presupuesto participativo en el Distrito } \\
\text { de Cartagena. }\end{array}$ & $\begin{array}{l}\text { Secretaria del Interior y } \\
\text { Convivencia } \\
\text { Ciudadana. }\end{array}$ \\
\hline
\end{tabular}

Fuente: Alcaldía de Cartagena, 2020.

6. Capítulo 4: Propuesta incluyente para la generación de una cultura de gestión participativa, para el mejoramiento del proceso de implementación del presupuesto participativo en la ciudad de Cartagena de Indias.

Luego de haber recorrido el marco jurídico que ampara el presupuesto participativo y de haber examinado cuáles son las problemáticas que obstaculizan la implementación del mismo, se procede a plantear una estrategia que propenda por que los cartageneros se vuelvan activos, se involucren y se apropien de este proceso, y de igual manera que demanden a través de las vías legales la puesta en marcha del PP y como se ha venido mencionando la ejecución de lo expuesto en los planes de desarrollo distritales.

Lo principal es centrarse en la adopción de una estrategia incluyente para la implementación y ejecución del Presupuesto Participativo, para esto, es fundamental establecer que un PP es un mecanismo acordado entre las dos partes- el gobierno local y los ciudadanos del territorio- de participación social y una herramienta de gestión local que posibilita la creación en conjunto del desarrollo humano sostenible conjuntamente, y en esa medida esta estrategia debe poseer dos objetivos primarios.

El primero debe girar en torno a la adopción de la Metodología del Presupuesto Participativo como mecanismo efectivo de Participación Ciudadana y Control Social de manera obligatoria y permanente, esto en concordancia con lo estipulado en el artículo 40 de la Ley 1551 del 2012. Dicha metodología permitirá a los ciudadanos y a la comunidad estar informados, intervenir, decidir, realizar veedurías y evaluar la gestión de las inversiones y obras que se ejecuten con recursos del rubro presupuestal denominado Presupuesto Participativo.
A partir de aquí la estrategia debe dejar en claro que los encargados de coordinar y organizar la Metodología del Presupuesto Participativo son: a) la Secretaría de Participación y Desarrollo Social; b) los Alcaldes Locales, c) la Secretaría de Hacienda. Serán ellos quienes realizarán las convocatorias a la comunidad para comenzar y finalizar el proceso en todas sus etapas. En ese orden de ideas, el proceso del Presupuesto Participativo se debe desarrollar contemplando las siguientes etapas:

1. Convocatoria: en esta etapa se deberá hacer un llamado abierto a todas las organizaciones sociales y ciudadanas que tenga cada localidad. Como fue mencionado, los responsables de tal acción serán las entidades encargadas, esto con el fin de que la ciudadanía participe en todo el proceso del Presupuesto Participativo e inscriban sus representantes en la Alcaldía Local.

2. Asambleas por Localidad: una vez se hayan inscrito y seleccionado los representantes de cada localidad de la ciudad, se realizará una reunión con el propósito de sensibilizar y educar sobre el contenido y los alcances de la Metodología del Presupuesto Participativo.

3. Asambleas por cada Unidad Comunera de Gobierno: en esta fase se llevarán a cabo diálogos deliberativos con cada Unidad Comunera de Gobierno que tengan por finalidad realizar los diagnósticos sociales y priorizar los proyectos de inversión. Cabe señalar que dentro de esta etapa se deben tener en cuenta las necesidades básicas insatisfechas de la comunidad, los objetivos del desarrollo trazados por la Organización de las Naciones Unidas y otros organismos internacionales, el Plan de Desarrollo Distrital y el Plan de Desarrollo de la Localidad. 
4. Aprobación de los programas y proyectos de inversión: una vez más se realizará una reunión con el ánimo de aprobar los programas y proyectos de inversión según lo discutido en el ejercicio de priorización. Es necesario señalar que éstos deben ser concertados por las comunidades en las Unidades Comuneras de Gobierno, por medio de Acuerdo de la Junta Administradora Local.

5. Contratación, ejecución y evaluación de los Proyectos de Inversión del Presupuesto Participativo: la contratación con las Organizaciones Comunales debe ser una realidad, debido a que, de conformidad con el artículo 55 de la Ley 743/02, el parágrafo 4 del artículo 6 de la Ley $1551 / 12$ y el artículo 68 de la Ley 1617/13, no menos del $30 \%$ de la contratación con recursos de los Fondos de Desarrollo Local y del Presupuesto Participativo deben ser adjudicados a las Organizaciones Comunales de la respectiva localidad.

El fin último es que esta metodología se presente ante el concejo distrital de Cartagena, para que se eleve a proyecto de acuerdo y sea obligatoria, so pena de que su incumplimiento genera una falta disciplinaria.

El segundo objetivo de la estrategia incluyente presentada en este documento es la activación de vías claras informativas sobre las problemáticas en las Unidades Comuneras de Gobierno mediante las Juntas de Acción comunal, para fomentar espacios donde se pueda visibilizar una verdadera cultura de gestión participativa y emplear las herramientas que proporciona la ley a sus ciudadanos, apropiarse de ellas y reclamar de manera clara los derechos fundamentales impuestos en la Constitución.

Entre otras vías de activación se encuentran diversos canales de comunicación como lo son: las redes sociales, los correos electrónicos, mensajes de texto e invitación por radio Am y Fm a participar de las instancias de gestión participativa.

La presente estrategia estará entrelazada con el uso del Modelo Integrado de Planeación y Gestión, que tiene como objetivo, la realización de la gestión institucional con principios de democracia participativa y democratización de la gestión pública. Esto acarrea la obligación de abrir espacios de participación e involucrar a los ciudadanos y las organizaciones de la sociedad civil en la formulación, ejecución, control y evaluación de la gestión pública.

\section{Conclusiones}

- Se puede denotar que el Presupuesto Participativo, si bien no cuenta con una ley específica que lo regule, goza de un amplio marco jurídico que lo acobija, y que además permite que la administración local implemente esta herramienta para poner en evidencia la importancia de la utilización de los mecanismos de participación ciudadana. Se hace la salvedad en cuanto a la corresponsabilidad legal que existe en este ámbito, es decir, es responsabilidad del Gobierno emplear el $\mathrm{PP}$, pero de igual forma es responsabilidad de los habitantes hacer uso de la normativa vigente para demandar el cumplimiento de la implementación total del presupuesto participativo.

- Cartagena carece de experiencia en la implementación del presupuesto participativo, puesto que la única experiencia fue llevada a cabo en 2009, posterior a eso, se realizó otro intento en 2016 y no fue exitoso, debido a la no realización de las asambleas que esta herramienta participativa necesita. Y en la actualidad, a pesar de estar implantado en el plan de desarrollo, las actividades para el mismo no han sido asignadas.

- En Cartagena se observan de manera clara las problemáticas que se encuentran relacionadas a la implementación del proceso de presupuesto participativo, entre los factores importantes están: la poca o nula asignación de recursos que requiere ejecutar este tipo de herramientas. Y la carencia del llamado a la ciudadanía por parte de las entidades territoriales, a participar en la toma de decisiones y el destino de los recursos distritales.

\section{Referencias}

Acuerdo Distrital 003 de 2009 (2009, 20 de marzo). Concejo Distrital de Cartagena.

Alcaldía Mayor de Bogotá, (2012). Plan de Desarrollo" Bogotá Humana". Plan de Desarrollo" Bogotá Humana, 2016. Recuperado de

http://www.sdp.gov.co/portal/page/portal/Porta ISDP/ciudadania/PlanesDesarrollo/BogotaH 
Alcaldía Mayor de Cartagena. (2016). Plan de Desarrollo" Primero la gente" 2016-2019. Recuperado de https://bicentenario.cartagena.gov.co/Document os/2016/Transparencia_y_aip/Plan_de_Desarr ollo/2016/ACUERDO-006-2016-PLAN-DEDESARROLLO.pdf

Alcaldía Mayor de Cartagena. (2020). Plan de Desarrollo "Salvemos Juntos a Cartagena" 2020-2023. Recuperado de http://ieu.unal.edu.co/images/Planes_de_Desarr ollo_2020/2.Plan_de_Desarrollo_Cartagena_2 020-2023.pdf

Becerra, P. (2017). Desarrollo del presupuesto participativo a nivel de Latinoamérica y de Colombia [Tesis de Magister en Derecho. Universidad Santo Tomás, Bucaramanga]. Archivo digital. https://repository.usta.edu.co/bitstream/handle/ 11634/3931/2017-

BecerraReyesPrudencioAntoniotrabajodegrado.pdf? sequence $=1 \&$ is Allowed $=y$

Betancourt, C. (2005) Presupuesto participativo en Colombia. Caracterización, evolución y perspectivas: revisión conceptual. Bogotá: ESAP Facultad de investigaciones.

Borda, F. (1998). La Grieta de la Democracia y la participación en Colombia.

Contreras, M. (2007). La Economía Política del Proceso Presupuestal en Colombia. Bogotá: Fedesarrollo - Documentos de Trabajo

García, R. (2003). Politicas Públicas Gobierno Local. Bogotá.

González, E. (2012). XVII Congreso Internacional del CLAD sobre la Reforma del Estado y de la Administración Pública, Cartagena, Colombia, 2012. Disponible en http://siare.clad.org/siare/abcd2/fulltext/con17/ gonzarod.pdf

http://www.secretariasenado.gov.co/senado/bas edoc/ley_0134_1994.html

Jiménez, R., Jiménez, I., y Alamilla, M. (2013). La importancia del proceso de planificación y el presupuesto administrativo en las instituciones gubernamentales. XIKUA Boletín Científico de la Escuela Superior de Tlahuelilpan. 1. 10.29057/xikua.v1i2.1192.

Ley 134 de 1994. (1994, 31 de mayo). Congreso de la República de Colombia. Diario Oficial 41.373

Ley 1551 de 2012. (2012, 6 de julio). Congreso de la República de Colombia. Diario Oficial No. 48.483 https://www.redjurista.com/Documents/ley_155 1_de_2012_congreso_de_la_republica.aspx\#l

Ley 1757 de 2015. (2015, 6 de julio). Congreso de la República de Colombia. https://www. funcionpublica.gov.coleva/gestorno rmativo/norma.php?i=65335

Mairal, A. (1998). Descentralización, regionalización y autonomía local. Universidad Nacional de Colombia. Colombia.

Pagliai, C. y Montecinos, E. (2006) Presupuestos participativos. Chile: Experiencias y aprendizajes. Recuperado de http:/library.fes.delpdffiles/bueros/chile/04624.pdf

Sánchez, F. L. (2004). Los presupuestos participativos: nuevos mecanismos de innovación democrática en los gobiernos locales Participative budget: A new tool for democratic innovation in local government. Intervención Psicosocial, 13(3), 325-344. Recuperado de http://www.copmadrid.org/webcopm/publicacio nes/social/93335.pdf

Subirats, J. (2005). Democracia, participación y transformación social. Polis Revista latinoamericana, Vol. 4, No. 12. Recuperado de https://dialnet.unirioja.es/servlet/articulo?codig $\underline{o=1350652}$

Tabares, A (2014). Afectación del ejercicio de presupuesto participativo de 2012 en el presupuesto de la localidad de Engativá, Bogotá D.C., en la vigencia 2013. Tesis de especialización en finanzas y administración pública. Universidad Militar Nueva Granada. Bogotá, Colombia. 
Urán, O. A. (2007) La participación ciudadana en la planeación y financiamiento de la ciudad como institución democrática emergente. Los casos de Manchester, Medellín y Porto Alegre. Revista Controversia, (189), 173-211. 